\title{
Health Psychology and Cardiovascular Disease Research Models
}

\author{
Raúl Martínez Mir* \\ Department of Psychology and Communication, University of Sonora, Mexico
}

*Corresponding author: Raúl Martínez Mir, Department of Psychology and Communication. University of Sonora, Blvd. Luis Encinas y Rosales s.n., Hermosillo, Sonora, México.

To Cite This Article: Raúl Martínez Mir.Health Psychology and Cardiovascular Disease Research Models. Am J Biomed Sci \& Res. 2019 - 6(2). AJBSR. MS.ID.001014. DOI: 10.34297/AJBSR.2019.06.001014

Received: 眥 November 8, 2019; Published: 眥 November 14, 2019

\section{Introduction}

Health psychology focuses its attention on diseases where the behavior is part of the origin, or the aggravation of them. It gives much importance to the effect that behavior has on the disease and therefore it is a discipline that complements the biomedical model of diseases.

Understanding the origin of the disease, the factors that protect it from its appearance (protectors), and the factors that influence its appearance (risk factors) is vital to understand health promotion, prevention and the treatment to be successful.

Cardiovascular diseases are the leading cause of death in industrialized countries. The study of the risk factors of this constant disease that sedentary lifestyle, obesity, alcohol and tobacco consumption, among others, are factors that make the occurrence of this disease more likely. However, when research models try to explain the origin of this disease from these factors, they do not reach more than $50 \%$. It was two eminent cardiologists Dr. Ray Rosenman and Dr. Meyer Friedman who were late in the fifties, observed a pattern of behavior in their patients, aimed at constant competitiveness, with high hostile responses and clinical and defensive thoughts, They called this Type A Behavior Pattern (TABP) [1]. From that moment on, the investigations expanded to include emotional, cognitive and behavioral aspects in the study of disease in general and cardiovascular disease.

Subsequent studies have suffered the same risk, and not all components of PCTA were equally harmful and harmful to the disease, noting that hostility was the most negative element of PCTA. Redirecting studies to hostility, its behavioral component aggression, and its emotional component anger. The studies were very promising, and even now they are taking these variables into account in the research.
It should be noted that although research considers the psychological phenomenon as part of the study of these diseases, and many others, in most cases this type of studies suffers from an adequate interpretation of psychological results, or there are simply no health psychologists involved in them. An example, following the issue of cardiovascular diseases, is the measurement of the hostility construct, which does not continue making instruments with the HO questionnaire prepared by Cook \& Medley [2] that was extracted directly from the Minnesota Inventory of multiphasic personality, and that has demonstrated in multiple investigations that it is an inconsistent instrument and does not adhere to any explanatory model of hostility, or instrument such as the Jenkins Activity Survey [3] closer to a psychological vision but also shows deficiencies.

From the author's point of view, it is necessary to consider psychological models of aggression and their relationship with the physiological response, as well as the effect of stress on the cardiovascular system. For this, it is necessary, in addition to knowing the model, to have a broader vision that develops the vision of a normal behavior and subsequently a pathological behavior, so that the contribution of the psychologist in this research protocol does not have to be a simple appearance in a instrument that measures a psychological variable, but the integration of results, biological variables, psychological variables, interconductal history of the individual and microsocial and macrosocial environment, which is capable of integrating an explanatory model where all these variables explain their behavior and effect About the disease being studied.

Examples of this are the biological health model of Emilio Ribes [4], as well as the strategic variations by Julio A Piña [5] on the previous model. Where the situations related to stress and the history of the subject's competences are integrated, reasons for 
carrying out the actions, biological events that have affected the individual, as well as biological function, social dispositional events, and competencies of the individual, all these elements will lead the subject to have protective or risk behaviors that will probabilism the genesis of the diseases to a greater or lesser extent.

Subsequently, the psycho-emotional effect of this disease on the individual must be considered to assess adherence to medical treatment, and consequently the effects that it must produce. Therefore, considering all the above is to consider the importance of a bio-psycho-social approach, where all components are considered of equal relevance, and not just a biologically approximation. that includes psychological variables that have little or nothing to do with explanatory models of the origin of the disease.

\section{References}

1. Rosenman RH, Friedman M (1961) Association of a specific behavior pattern in women with blood and cardiovascular findings. Circulation 24: $1173-1184$

2. Cook WW, Medley DM (1954) Proposed hostility and pharisaic-virtue scales for the MMPI. J Appl Psychol 38: 414-418.

3. Jenkins CD, Zyzanski SJ, Rosenman RH (1979) The Jenkins Activity Survey. Nueva York: Psychological Corporation.

4. Ribes-Iñesta E (1990) Psychology and Health A conceptual analysis. Barcelona: Martínez-Roca.

5. Piña López JA (2008) Variations on the psychological model of biological health of ribes: Justification and development. Univ Psychol 7(1): 19-32. 